

\section{polisemie}

\section{I}

\section{1}

Direzione

Stefano Milonia (University of Warwick)

Comitato scientifico

Giulia Bassi (Università degli Studi di Siena)

Mario Cianfoni (Sapienza Università di Roma)

Stefano Colangelo (Alma Mater Studiorum - Università degli Studi di Bologna)

Luigi Marinelli (Sapienza Università di Roma)

Carlo Pulsoni (Università degli Studi di Perugia)

Niccolò Scaffai (Università degli Studi di Siena)

Costantino Turchi (Sapienza Università di Roma)

Fabio Zinelli (École Pratique des Hautes Études)

\section{Redazione}

Stefano Bottero (Università Ca' Foscari Venezia)

Mattia Caponi (Sapienza Università di Roma)

Carlo Londero (Università degli Studi di Udine)

Giorgio Tranchida (Alma Mater Studiorum - Università degli Studi di Bologna)

Samuele Maria Visalli (Sapienza Università di Roma)

Arianna Saggio (Sapienza Università di Roma)

Andrea Bongiorno (Aix-Marseille Université)

Giulia Boitani (University of Cambridge)

Alessandra Frustaci

Polisemie è una rivista annuale pubblicata dalla University of Warwick Press.

Gli articoli pubblicati nella sezione Saggi sono sottoposti a double-blind peer review.

Licenza Creative Commons - Attribuzione (CC-BY 4.0).

ISSN: 2634-1867

DOI: 10.31273 /polisemie.v1

Immagine copertina: Serse Luigetti 


\title{
LA FUNZIONE DELLA NOSTALGIA CREATIVA
}

\author{
Giulia Martini
}

Scilla e Cariddi uccidono in due modi opposti: per selezione, con specificità micidiale, e per grossolanità, con larghissimo ingurgito. Queste sembrano le due morti in cui rischia di incorrere anche il gesto antologico; soprattutto quando il gesto antologico diventa un gesto relazionale, un tendersi la mano per rimanere in simil-equilibrio, sui flutti mossi delle emersioni individuali e del coacervo.

Ma tra individualismo e coacervo è possibile operare una scelta, ritagliare il mondo, tentare una ricognizione, per quanto minacciata dai pericoli della vicinanza e dall'azzardo: un accertamento dell'esistenza e dell'identità di una generazione, attraverso una constatazione diretta, una campionatura strategica delle risorse tematiche e stilistiche che vengono dispiegate.

A partire dal corpus dei ventiquattro autori presenti nell'antologia Poeti italiani nati negli anni '80 e '90 (Interno Poesia, I vol. 2019, II vol. 2020), ${ }^{1}$ questo lavoro si propone di focalizzare e mettere in relazione i nodi tematici ricorrenti nei testi. Per ogni tema verranno offerti esempi testuali (non più di quattro e non meno di due per ogni autore, per ragioni di spazio) selezionati dal ventaglio molto più ampio dei due volumi antologici, a cui si rimanda per un quadro esaustivo.

Per anticipare un primo approdo, dall'inquadramento critico dell'incrocio dei temi emersi a posteriori sembrerebbe che i ventiquattro autori presi come campione partecipino orizzontalmente all'istanza della nostalgia creativa ${ }^{2}$ e che il

\footnotetext{
${ }^{1}$ Giulia Martini (a cura di), Poeti italiani nati negli anni ' 80 e '90, in due voll., Latiano, Interno Poesia, 2019-2020, d'ora in avanti Poeti italiani. I due volumi ospitano testi di (in ordine alfabetico): Maddalena Bergamin, Ophelia Borghesan, Maria Borio, Clery Celeste, Marco Corsi, Damiana De Gennaro, Tommaso Di Dio, Gianluca Fùrnari, Manuel Giacometti, Anita Guarino, Giovanni Ibello, Marco Malvestio, Franca Mancinelli, Lorenzo Marinucci, Demetrio Marra, Dimitri Milleri, Giusi Montali, Francesco Ottonello, Bernardo Pacini, Mariachiara Rafaiani, Eleonora Rimolo, Damiano Sinfonico, Francesco Terzago, Francesco Vasarri.

${ }^{2}$ Nell'accezione di Remo Ceserani, Mario Domenichelli, Pino Fasano, Dizionario dei temi letterari, vol. II, Milano, Garzanti, 2007, p. 1669. Di «nostalgia creativa» parla anche Vito Teti, in Antropologia dei migranti, in La storia, le trasformazioni. Piero Bevilacqua e la critica del presente, a cura di Leandra D'Antone e Marta Petrusewicz, Roma, Donzelli, 2015. Un'altra fonte è Emilia Di Rocco, in particolare lo studio Raccontare il ritorno. Temi e trame della letteratura, Bologna, il
} 
movimento interno dei loro testi indichi, a vario livello, una trasformazione della nostalgia passiva in spinta narrativa e propensione all'agency, attraverso la presa di consapevolezza della disgregazione del mondo e della cellula sociale.

Parte di questi temi era in qualche modo emersa già nella Prefazione di entrambi i volumi, in cui si accennava a una sorta di «attivismo non contemplativo ${ }^{3}{ }^{3}$ come a una postura testuale comune individuata solo a posteriori; in altre parole, senza che abbia orientato in alcun modo la scelta dei poeti antologizzati. Con questo articolo si vorrebbe quindi circoscrivere e approfondire a un livello razionale e oggettivo quanto nelle introduzioni era stato proposto a un livello intuitivo.

\section{Alcuni temi e oggetti poetici degli anni Venti}

Fin da subito emerge, legata alla ricorrenza orizzontale e sistematica di mezzi di trasporto (i più distanti fra loro, da una «ruspa» a un'«arca», in linea con le differenze stilistiche e programmatiche di ciascun autore), l'ossessione condivisa per la partenza, che si configura tanto nei termini di viaggio compartecipato che in quelli di spinta individuale.

Nel primo caso, il personaggio che dice «io» contempla i viaggi degli altri, magari da un punto di vista isolato, oppure si trova in un mezzo, pubblico o privato, con altre persone, e il viaggio è uno spostamento condiviso, un'esperienza comunitaria o familiare in senso stretto: «aspettiamo che un sessantenne | meccanico [...] | aggiusti la nostra Renault sette posti»; $;^{4}$ «Ho questo amico con cui spesso facciamo dei giri $\mid$ in macchina, giriamo la città.$^{5}$

Nel secondo, la partenza è legata a un'avventura, anche quotidiana ma comunque personale, alla scoperta dell'eros, e, finalmente, al nostos, al ritorno a casa: «il bus invisibile | che traghetta a casa». 6

L'occasione del viaggio dà adito talvolta a una partenza assoluta, quasi senza soggetto, che può avere esiti di ansia religiosa, per cui il mezzo di trasporto viene connotato con elementi sovrannaturali o sublimato a 'macchina' per eccellenza, a motore universale: «L'incanto di una teleferica $;^{7}$ «le macchine in processione». ${ }^{8}$

Sembra altrettanto interessante che questa partenza non prenda mai in considerazione l'idea della fuga: nessun mezzo di trasporto viene preso per fuggire,

Mulino, 2017. Sul rapporto tra forze creative e crisi, si veda anche Evelyn Grossman, La Creativité de la crise, Paris, Les Éditions de Minuit, 2020.

${ }^{3}$ Poeti italiani, I, p. 6.

${ }^{4}$ Ogni giardino è un poliambulatorio, vv. 3-5, D. Marra, Poeti italiani, I, p. 155.

${ }^{5}$ Ho questo amico con cui spesso facciamo dei giri, vv. 1-2, Terzago, Poeti italiani, II, p. 96.

${ }^{6}$ Quando si abita il panico, vv. 4-5, Milleri, Poeti italiani, I, p. 167.

${ }^{7}$ La mia estasi rimane, v. 8, Ibello, Poeti italiani, I, p. 108.

${ }^{8}$ La funzione non è durata molto, v. 2, Sinfonico, Poeti italiani, I, p. 82. 
per allontanarsi velocemente da qualcosa o qualcuno. Il viaggio, in altre parole, viene intrapreso per capire o per avvicinarsi («Sapersi avvicinare. | Così vediamo l'enigma della distanza $\mid$ dal posto in cui si addensano i luoghi che ci hanno abitato»), ${ }^{9}$ primo dato per cui questo manipolo di autori condividerebbe la postura della propensione e della contemplazione industriosa.

Fra le varianti del macrotema del viaggio, la più ricorrente all'interno della selezione operata è quella del nostos, del ritorno a casa. Il concetto di casa, a sua volta, indica tanto il luogo fisico dove sono state erette le mura domestiche quanto il momento ideale del ricongiungimento degli affetti del presente e del passato, e infine la presa di consapevolezza rispetto al proprio percorso di individuazione.

Di nuovo, ci troviamo di fronte a due istanze contrapposte: da un lato, questo amalgama di stati chiamato casa non è mai pacificato e neppure omogeneo, ma viene fatto reagire con le categorie della guerra, della distanza, del disorientamento e dell'abbandono; dall'altro, anche in questo caso emerge una propensione a tornare che dipende strettamente dall'agency dell'individuo: «Le case non sono mai belle $\mid$ come quando le abbandoniamo $\gg ;{ }^{10} \ll$ È una trincea la casa materna dove mi ospiti». ${ }^{11}$

Lo sfaldamento o contaminazione del nucleo originale è un sintomo del più grande sfaldamento o contaminazione del mondo, che ricorre orizzontalmente in tutti gli autori presi come campione. Niente viene esonerato da questa disgregazione generalizzata, che coinvolge tanto l'individuo, consapevole di avere «le radici nel flusso del nulla», ${ }^{12}$ quanto la famiglia, ${ }^{13}$ il consorzio sociale ${ }^{14}$ e il paesaggio, ${ }^{15}$ nelle forme ricorrenti del crollo o caduta e del malfunzionamento:

\footnotetext{
${ }^{9}$ Sapersi avvicinare, vv. 1-3, Borio, Poeti italiani, I, p. 31.

${ }^{10}$ Say Goodbye, Catullus, to the Shores of Asia Minor, II, vv. 21-22, Malvestio, Poeti italiani, II, p. 131.

${ }^{11}$ Quell'abbraccio te lo sei preso tutto, v. 4, Rimolo, Poeti italiani, I, p. 128.

${ }^{12}$ Jetzt, e quali fonti Neubauer, v. 8, Ottonello, Poeti italiani, II, p. 157.

${ }^{13}$ «Il padre conquista | e poi scompare, | si duplica e fugge. [...] La figlia è remissiva, dolente. [...] si logora nel nascondino. [...] sognare un parricidio | che investa tutto il futuro, | tutto il passato» (Ritratto di famiglia, vv. 1-21, Milleri, Poeti italiani, I, p. 168).

${ }^{14}$ «Avevo degli amici bellissimi, a vent'anni, | icone o stereotipi da film. | Diventavano tanti volti sfaccettati, ubriachi ed ostili» (Avevo degli amici bellissimi, a vent'anni, vv. 1-3 Guarino, Poeti italiani, I, p. 21).

15 «Stanno | erigendo delle nuove palazzine, mi fa notare il mio amico. | Hanno spianato tutto quanto, hanno scavato delle fosse. | Hanno bruciato gli ultimi sterpeti. Stanno combattendo | il terzo paesaggio» (Ho questo amico con cui spesso facciamo dei giri, vv. 11-15, Terzago, Poeti italiani, II, p. 96).
} 
la lingua che si inceppa è un chiaro segno ${ }^{16}$

il caro corpo cade ${ }^{17}$

Se adesso arrivasse un terremoto,

saremmo tutti insieme $e^{18}$

Le frasi non compiute restano ruderi. C’è un intero paese in pericolo di crollo ${ }^{19}$

Dentro la vita morbida ci si gettava il seme

del futuro, ma nulla funzionava ${ }^{20}$

Questo deterioramento universale sembra applicato in particolare a due sottocategorie: il cibo e il corpo. Nel caso del cibo, assistiamo a un progressivo snaturamento di tutto ciò che avrebbe potuto essere nutriente. Il cibo diventa una minaccia, è sempre nauseante e respingente, quando non acido e aspro, ostile o giudicante, avariato, acquista lo statuto di un'arma, di un potenziale veleno, di un proiettile, con risvolti inquietanti in senso metafisico:

Sabato frittole e curcuci. Nelle caddàre vengono bolliti dalla mattina gli scarti del maiale ${ }^{21}$

il caffe torrefatto sciama dentro

l'abisso

[...] al finire della maionese, ho paura di morire ${ }^{22}$

il latte, nelle bottiglie verdi, ha lasciato una crosta gialla, appiccicosa ${ }^{23}$

In tutti questi casi, si tratta di cibo finto, cibo che non nutre, e se nutre, è poco e sta per finire.

\footnotetext{
${ }^{16}$ Raggiunge il terzo cielo, quindi pensa, v. 3, Borghesan, Poeti italiani, II, p. 115.

${ }^{17}$ un diagramma cosi, Corsi, Poeti italiani, II, p. 45.

${ }^{18}$ Se adesso arrivasse un terremoto, vv. 1-2, De Gennaro, Poeti italiani, I, p. 139.

${ }^{19}$ Le frasi non compiute restano ruderi, Mancinelli, Poeti italiani, II, p. 20.

${ }^{20}$ Le case crolleranno, si è dimezzato, vv. 14-15, Vasarri, Poeti italiani, I, p. 96.

${ }^{21}$ La vita a Kansas City, VI, vv. 1-3, Marra, Poeti italiani, I, p. 152.

${ }^{22}$ Senza sarcasmo, vv. 15-23, Pacini, Poeti italiani, I, pp. 65-66.

${ }^{23}$ Ho questo amico con cui spesso facciamo dei giri, vv. 16-17, Terzago, Poeti italiani, II, p. 96.
} 
Passando invece al corpo, il suo impiego come oggetto testuale percorre entrambi i crinali della repulsione e della violenza sanguinosa, sempre a partire da un contesto generale di frantumazione e sgretolamento:

Il tuorlo magmatico dell'alba si sgretola nei cardi.

È questo il destino dei corpi ${ }^{24}$

l'idrofobia, il cane che beve, il corpo che si disgrega ${ }^{25}$

il nostro naturale

decomporci in schegge di terra ${ }^{26}$

Per quanto riguarda la repulsione, i ventiquattro autori antologizzati fanno del corpo un ricettacolo di malattie infettive, virali, verminose, sindromi, non necessariamente terminali, ma sempre fastidiose e respingenti, che deturpano l'aspetto e ottundono i sensi. ${ }^{27}$ Queste malattie possono riguardare tanto la pelle quanto la psiche, ${ }^{28}$ nei termini di nevrosi o disturbo della personalità, ${ }^{29}$ con esiti invalidanti.

In una nutrita serie di testi, assistiamo a scene che rispondono a un gusto decisamente splatter, per cui vengono evocate situazioni emorragiche, orrende mutilazioni e ferite mortali, magari volontariamente inferte, che implicano la morte di un congiunto del soggetto parlante: «ti si spaccavano i pori $;^{30} \ll$ rotto il tempo e le vene del collo». ${ }^{31}$

Spesso si tratta di una morte generica, di un individuo eletto campione della specie, sia attuale che di un lontano passato; senza escludere le morti mediatiche: una vittima della malavita organizzata e un suicidio famoso: $\ll$ (La Fallara $\mid$ solo un

${ }^{24}$ Il tuorlo magmatico dell'alba, vv. 1-3, Ibello, Poeti italiani, I, p. 107.

${ }^{25}$ i portatori di peste - io che mi sdraio e mi immagino il tuffo, v. 6, Montali, Poeti italiani, II, p. 85.

${ }^{26}$ Sofiya, vv. 10-11, Rimolo, Poeti italiani, I, p. 126.

$27 \ll$ Malattia infettiva generalizzata | con sintomatologia caratteristica» (Setticemia, vv. 1-2, Bergamin, Poeti italiani, II, p. 61).

${ }^{28}$ «La mattina, per lei, il referto ha usato una parola | rocciosa, senza pori: depressione, cadere verso il basso» (Anedonia, vv. 15-16, Borio, Poeti italiani, I, p. 39).

${ }^{29} \ll \mathrm{Ha}$ chiesto se il braccio era il suo» (Ha firmato lentamente il modulo del consenso, v. 5, Celeste, Poeti italiani, I, p. 118); «la ciclotimia è l'ultima fiducia della specie» (il corpo si avvia, proietta luce opaca, v. 10, Montali, Poeti italiani, II, p. 86); «le braccia insugherite» (Senza sarcasmo, v. 8, Pacini, Poeti italiani, I, p. 65).

${ }^{30}$ Le case crolleranno, si è dimezzato, v. 21, Vasarri, Poeti italiani, I, p. 96.

${ }^{31}$ Tombali in catalogo, v. 72, Giacometti, Poeti italiani, I, p. 52. 
po' d'acidità di stomaco) $\gg ;^{32}$ «Morire dal ponte, passati i cartelli $\mid$ varcare i cancelli e buttare | la tenera mente in altissime | acque ghiacciate $\gg .{ }^{33}$

Talvolta, chi scrive si preoccupa di contestualizzare la morte in senso luminoso, cioè di fornire indicazioni circa lo stato della luce nel momento in cui avviene il decesso. ${ }^{34}$

Questo permette di introdurre un altro oggetto imprescindibile, la luce, onnipresente e ossessivamente misurata: «e luce dappertutto, ancora luce» $;^{35}$ «Non vedevi $\mid$ come tutto luccicava». ${ }^{36}$

Rispetto alla mera registrazione della cornice luminosa, variabilmente intersecata tra i due estremi di oscurità totale e abbaglio, emerge un rapporto di causalità materiale che viene a crearsi fra l'azione o l'evento evocato e l'ora del giorno. ${ }^{37}$ Non di rado, la luce subisce un processo di personificazione che la porta a compiere un gesto proprio, posizionandosi quasi come un personaggio. ${ }^{38} \mathrm{Se} \mathrm{da}$ un lato non mancano annotazioni e metafore classiche o classicheggianti, dall'altro il dato luminoso viene riformulato in immagini rare, preziose e ricercate, facendo ricorso a una terminologia settoriale o straniante. ${ }^{39}$

Una prima domanda riguarda il rapporto tra il soggetto parlante e la luce. Dalla comparazione delle ricorrenze è emerso che chi scrive assume nei confronti della luce la postura di chi attende per agire; il soggetto lirico sembra avere una sorta di appuntamento luminoso, aspetta fiducioso di 'venire alla luce', in linea con la costante della propensione, con la lotta contro il buio di cui sopra: «Il primo appuntamento fu alla luce $\mid$ nell'ora della luce $\gg ;^{40}$ «Aspetto che scenda la luce $\gg .^{41}$

In tal senso, una conseguenza interessante è quella del coincidere del soggetto parlante (del suo corpo, del suo conglomerato identitario o delle sue ipostasi) con la fonte luminosa; chi dice «io» diventa tramite di luminosità, proietta luce: «un solo punto luce che sono io»; ${ }^{42} \ll$ la coclea $\mid$ proietta un sole ${ }^{43}$

${ }^{32}$ La vita a Kansas City, VII, vv. 1-2, Marra, Poeti italiani, I, p. 152.

${ }^{33}$ Forth Road Bridge, vv. 6-9, Bergamin, Poeti italiani, II, p. 60.

34 «la morte col sole che splende» (Ivi, v. 2).

${ }^{35}$ Le nocche come acini sbucciati, v. 7, Borghesan, Poeti italiani, II, p. 111.

${ }^{36}$ è un momento di ammutinamento, vv. 9-10, Rafaiani, Poeti italiani, II, p. 167.

37 «nell'ora chiara della ricerca del cibo» (A ottanta centimetri da te, v. 5, Celeste, Poeti italiani, I, p. 116).

38 «la poca luce | emise un breve rantolo» (fissavo l'ombra sul muro e per esercizio, vv. 11-12, Corsi, Poeti italiani, II, p. 46).

39 «il sole è una biglia di benzodiazepina» (Cercava la risacca nelle pinete, v. 8, Ibello, Poeti italiani, I, p. 109).

${ }^{40}$ Il primo appuntamento fu alla luce, vv. 1-2, Fùrnari, Poeti italiani, II, p. 141.

${ }^{41}$ Aspetto che scenda la luce, Mancinelli, Poeti italiani, II, p. 21.

${ }^{42}$ Trasparenza, IV, v. 8, Borio, Poeti italiani, I, p. 35.

${ }^{43}$ se ne sta immobile, si muove invece l'occhio, vv. 2-3, Montali, Poeti italiani, II, p. 84. 
Il coinvolgimento diretto all'interno della fenomenologia della luce, il farsi testimone luminoso, sembra direttamente correlato a quella che risulta l'istanza principale tra quelle finora esposte: l'autoinvestitura poetica, che si risolve nei termini di progressiva specializzazione letteraria e presa di consapevolezza della specificità del gesto artistico, percepito come «mestiere»e «lavoro» da cui non bisogna distrarsi, anche quando si realizza nei termini di performance, di esibizione davanti a un pubblico:

Occorreranno tecnici del canto quando ogni altro mestiere avrà fallito ${ }^{44}$

Tutto era già pronto per il reading di poesie ${ }^{45}$

Per sopravvivere è necessario specializzarsi, diventare un esperto in qualche specie naturale, linguistica o sociale ${ }^{46}$

Ora il mestiere è non perdere il punto. Il lavoro è svagato, precisissimo, non si debbono perderne gli appunti ${ }^{47}$

Veramente colpisce la sicurezza per cui il poeta sa di fare poesia e forse, ancora di più, la disinvoltura con cui lo dice, insieme all'insofferenza per le «frasi non compiute ${ }^{48}$ o interrotte, per le «frasi sempre uguali ${ }^{49}$ per il «linguaggio mai perfezionato $\gg::^{50}$

Cosi, non alzando la voce per non essere scoperta, mi è riuscito finalmente di sembrare una poetessa ${ }^{51}$

\footnotetext{
${ }^{44}$ Occorreranno tecnici del canto, vv. 1-2, Fùrnari, Poeti italiani, II, p. 143.

${ }^{45}$ Tutto era già pronto per il reading di poesie, v. 1, Guarino, Poeti italiani, I, p. 17.

${ }^{46}$ Per sopravvivere è necessario specializzarsi, vv. 1-3, Sinfonico, Poeti italiani, I, p. 83.

${ }^{47}$ Appunti sul raddoppio, vv. 20-22, Vasarri, Poeti italiani, I, p. 94.

${ }^{48}$ Le frasi non compiute restano ruderi, Mancinelli, Poeti italiani, II, p. 20.

${ }^{49}$ Il lavoro è ricominciare a cicli, v. 6, Celeste, Poeti italiani, I, p. 117.

${ }^{50}$ L'opera della luce sugli stagni, v. 5, Fùrnari, Poeti italiani, II, p. 142.

${ }^{51}$ Ti ho tenuto il posto, vv. 8-11, Bergamin, Poeti italiani, II, p. 59.
} 


\begin{abstract}
Avremo scritto cento poesie sul canile prima di rimuoverle tutte insieme ${ }^{52}$

ora che scrivo ${ }^{53}$

scrivevo scevro in poesia ${ }^{54}$
\end{abstract}

Così, anche una parola quasi impudica come 'ispirazione', quasi blasfema come 'musa', può essere allusa con sfacciataggine, e proprio riferita al momento in cui «morde nel letto $\gg .{ }^{55}$ Anche quando il ruolo di poeta viene coinvolto testualmente in una scena declassante, sembra comunque una posa, un concedersi una postura auto-parodica, non scevra di compiacimento, che non arriva a intaccare la concezione dignitosissima della propria veste sociale; concezione che sembra motivata, più di ogni altra cosa, dal fatto di avere qualcosa da dire e di sapere come farlo, con mezzi stilistici e retorici precisi e ineliminabili:

non scrivere, davvero, lascia stare, ti stanno raccontando che là fuori

qualcuno vuole leggerti, ma è falso ${ }^{56}$

Lui non scandiva i momenti e gli spettatori si distraevano e ricominciava a diffondersi il vociare di frasi sconnesse.

Il Poeta aspettava, seduto, paziente, il suo turno tra

i frammenti dei discorsi che udiva.

Poi si alzò e diffuse il suono dei suoi versi nell'ambiente.

La sua presenza solida e la voce più

sicura riuscirono a zittire il pubblico insensibile $e^{57}$

ma a noi, stanchi e verbosi,

sempre più stanchi e sempre più verbosi,

non può che fare piacere ${ }^{58}$

\footnotetext{
${ }^{52}$ Canile, 100, vv. 1-3, Borghesan, Poeti italiani, II, p. 113.

${ }^{53}$ Ma fratello impossibile, mio solo, v. 6, Fùrnari, Poeti italiani, II, p. 144.

${ }^{54}$ Ricordo dopo la partenza, v. 12, Ottonello, Poeti italiani, II, p. 156.

${ }^{55}$ Se appaio cosi ordinata, ordinaria, è per il rispetto che provo, v. 6, Guarino, Poeti italiani, I, p. 23.

${ }^{56}$ Sei li che fai la spesa, che fai spinning, vv. 5-7, Borghesan, Poeti italiani, II, p. 112.

${ }^{57}$ Tutto era già pronto per il reading di poesie, vv. 9-15, Guarino, Poeti italiani, I, p. 17.

${ }^{58}$ Say Goodbye, Catullus, to the Shores of Asia Minor, vv. 12-14, Malvestio, Poeti italiani, II, p. 131.
} 
Sotto io, come dei cipressi erbacce, poeta a me rimane la scatarrata di chi ha macchiato il caffe con la schiuma di latte ${ }^{59}$

saprei cosa dire ma scatta il verde ${ }^{60}$

Questi mezzi stilistici e retorici possono all'occorrenza trasformarsi quasi in strumenti di tortura: succede quando l'atto del dire inizia a essere percepito come «crimine», ${ }^{61}$ quando il dire diventa giocoforza un «dire Orrore». ${ }^{62}$ Eppure, non basta per rinunciare all'enunciazione: la disgregazione del mondo non fa desistere dal dire, ma lo incentiva.

A tacere, semmai, è il referente, che evidentemente non possiede gli strumenti per riscattare il suo messaggio dall'afasia - tanto che viene ammonito da chi dice «io», che gli sconsiglia di parlare, gli consiglia di astenersi: «e io al contrario ho ancora $\mid$ qualcosa da dirti, vedi, nonostante tutto, $\mid$ cara $\gg .{ }^{63}$

Per contro, sono numerosi gli esempi in cui il poeta si immortala nell'atto di dire parole al suo interlocutore, parole che hanno sempre - o quasi - un ruolo chiarificatore, se non proprio salvifico, e che vengono lanciate quasi come una corda cui aggrapparsi («ti ho | gettato una corda di parolette sottili»). ${ }^{64}$

A questo proposito, sembra opportuno soffermarsi sui modi in cui ci si rivolge ai referenti. Ai ventiquattro autori antologizzati sembra infatti ascrivibile la dote della generosità interlocutoria, che prevede uno sforzo di apparato, uno zelo raro, nella direzione della dicibilità: nel momento in cui, come si è detto, esiste un contenuto, è importante che l'Altro lo capisca. Ecco allora prendere piede intercalari e frammenti di enunciato che indicano il messaggio e lo incorniciano, accentrano l'attenzione dell'interlocutore: «Capisci, non è stato per indifferenza o durezza di cuore».$^{65}$

Una variante abbastanza comune di questa premura nei confronti del referente è rappresentata da tutta una serie di testi in cui compaiono direttamente consigli e ammonizioni, (molto spesso nella forma di imperative costruite con l'infinito o

${ }^{59}$ Ogni giardino è un poliambulatorio, vv. 13-15, Marra, Poeti italiani, I, p. 155.

${ }^{60}$ Un limone, v. 10, Pacini, Poeti italiani, I, p. 68.

${ }^{61}$ Quando si abita il panico, v. 17, Milleri, Poeti italiani, I, p. 167.

${ }^{62}$ Ritratto di famiglia, v. 18, Milleri, Poeti italiani, I, p. 168.

${ }^{63}$ Ovidio in autostrada prima di un temporale lascia un messaggio in segreteria, vv. 17-19, Malvestio, Poeti italiani, II, p. 126.

${ }^{64}$ Le case crolleranno, si è dimezzato, vv. 18-19, Vasarri, Poeti italiani, I, p. 96.

${ }^{65}$ Mi hai telefonato mentre pensavo a Costanza d'Altavilla, v. 4, Sinfonico, Poeti italiani, I, p. 79. 
comandi negativi), delucidazioni e previsioni sul futuro: «Non dire bugie» $;^{66}$ «prova a dormire $\gg .{ }^{67}$

Questa pratica discorsiva comporta necessariamente che il personaggio parlante abbia una visione più completa, nitida, lungimirante delle cose - che, insomma, la sappia più lunga del suo interlocutore - e infatti, non di rado, viene ribadito quanto i loro posizionamenti nel mondo siano distanti, ${ }^{68} \mathrm{e}$, cosa ancora più interessante, quanto le risorse gnoseologiche (o anche semplicemente il carisma) dell'interlocutore siano limitate. ${ }^{69}$

La lungimiranza del soggetto lirico sembra andare di pari passo con un elemento formale che si candida a distinguere orizzontalmente la produzione giovanile degli autori scelti, pur non afferendo alle categorie di tema e oggetto testuale in senso stretto: l'uso - inconsapevole o programmatico (come nella serie Canile di Ophelia Borghesan),${ }^{70} \mathrm{ma}$ comunque sistematico e ricorrente - dell'indicativo futuro semplice come tempo verbale. ${ }^{71}$

Il testo poetico viene così caricato di un valore profetico, oltre a riconfermarsi anche grammaticalmente quella propensione che è già stata, più volte, guardata come caratterizzante in senso generazionale: sembra che questi autori abbiano continuamente lo sguardo rivolto al futuro - e non in atteggiamento solo contemplativo, ma in quello di chi si prepara ad agire, di chi aspetta la luce per farsi luce, per passare agli altri un testimone luminoso.

Semplificando, c'è un futuro luminoso (o presagito come tale) che viene edificato su un'eredità scomoda, quando non macabra, che affonda spesso le sue radici in un passato lontanissimo; che equivale ad alludere al frutto usando nel frattempo il cibo avariato come fertilizzante.

In molti testi si respira un'aria di caverna, come se fosse un recupero identitario, un «esercizio della ricostruzione $» ;^{72}$ si entra nei siti antichi nelle vesti di studiosi, restauratori, archeologi, speleologi o turisti $-\mathrm{e} \ll \operatorname{scava}[\mathrm{re}]$ le fondamenta», ${ }^{73}$

${ }^{66}$ Tombali in catalogo, v. 31, Giacometti, Poeti italiani, I, p. 50.

${ }^{67}$ Ukiware yo, Marinucci, Poeti italiani, II, p. 69.

${ }^{68}$ «Sono sempre altrove rispetto a te. E viceversa» (Avevo degli amici bellissimi, a vent'anni, v. 9, Guarino, Poeti italiani, I, p. 21).

${ }^{69}$ «non parli, immerso | nell'odore di urina e proteine animali» (Un uomo entra, vv. 9-10, Di Dio, Poeti italiani, II, p. 33).

${ }^{70}$ Cfr. Poeti italiani, II, p. 113.

${ }^{71} \ll \mathrm{Vi}$ aspetteremo, dentro il corpo $\mid$ del piccione sull'asfalto [...] $\mid$ A metà del viaggio, ci ritroveremo lungo il bordo | di un lago che vedremo | nella bugia della mente. Sapremo poi muoverci [...] | Sapremo parlare» (Infine si alzò dal tavolo, vv. 8-16, Di Dio, Poeti italiani, II, p. 34).

${ }^{72}$ A mani nude gli studiosi scavano le fondamenta, v. 5, Rimolo, Poeti italiani, I, p. 127.

${ }^{73}$ Ivi, v. 1. 
«trova[re] | residui organici» ${ }^{74}$ non è che un altro modo per ripetere il nostos, il rito del ritorno a casa:

Dentro la caverna, hanno trovato

residui organici, rocce e frammenti di corno

sbozzato in zagaglie $[\ldots]^{75}$

Necropoli aperte, cerose,

affastellate di restauratori

che cercano erose membra $[\ldots]^{76}$

tra tombe etrusche ${ }^{77}$

Gli studiosi le hanno dato il nome di Naia quando

la hanno ritrovata in un complesso di caverne dello

Yucatàn allagatosi circa diecimila anni $\mathrm{fa}[\ldots]^{78}$

\section{In dialogo}

Nell'ottica di mettere in relazione i nodi stilistici e tematici affiorati (e sono: i mezzi di trasporto, il nostos, la disgregazione del mondo, il cibo nocivo, le malattie repulsive, la luce, la generosità interlocutoria, l'uso del futuro semplice, l'eredità macabra e la resistenza archeologica), emerge come l'esperienza della nostalgia (nella cui etimologia si legano nostos e algos, dolore per la sua impossibilità) ricongiunga idealmente tanto la rappresentazione della malattia quanto il desiderio del ritorno a casa.

Nel Dizionario dei temi di Cesarani, la ricostruzione della storia letteraria della nostalgia si sviluppa a partire dalla sua origine come malattia (passata solo in un secondo momento a sentimento), quindi da una serie di informazioni di carattere medico, fra cui l'osservazione dei sintomi:

La parola nostalgia è moderna, ma il campo dei sentimenti che essa designa è antico quanto il linguaggio dell'uomo. La parola fu coniata soltanto nel 1688 da Johannes Hofer, un giovane studente di medicina dell'Università di Basilea, il quale intitolò la sua tesi di laurea Dissertatio medica de nostalgia. [...] Agli occhi dei medici di fine Seicento

\footnotetext{
${ }^{74}$ Un uomo entra, vv. 14-15, Di Dio, Poeti italiani, II, p. 33.

${ }^{75}$ Ivi, vv. 14-16.

${ }^{76}$ Tombali in catalogo, vv. 41-43, Giacometti, Poeti italiani, I, p. 50.

${ }^{77}$ Kami chisashi, Marinucci, Poeti italiani, II, p. 70.

${ }^{78}$ Gli studiosi le hanno dato il nome di Naia quando, vv. 1-3, Terzago, Poeti italiani, II, p. 98.
} 
i casi di nostalgia si mostrano con stati di febbre strisciante, allucinazioni, depressioni, circolazione lenta del sangue, nausea, disappetenza, insonnia, delirio: sintomi che il giovane Hofer spiega con la concentrazione degli «spiriti vitali» e degli umori attorno a una sola sede, il cervello, e attorno a una sola idea, il fantasma del ritorno in patria. ${ }^{79}$

Tra questi sintomi, come si è visto, almeno due interessano più o meno orizzontalmente la produzione degli autori analizzati come campione: la disappetenza e la nausea.

Per tornare al tema forte della loro poesia, il sentimento della disgregazione del mondo (molteplicemente declinato), sembra funzionare perfettamente la definizione di «nausea» offerta dallo stesso Dizionario dei temi:

Sono numerose le testimonianze di autori che reagiscono alla paralisi della volontà e al disgusto di fronte alla decomposizione della cellula sociale - sempre scanditi dalle guerre e dai loro esiti catastrofici -, con la rappresentazione di un sentimento di nausea nel soggetto. ${ }^{80}$

Inoltre, in Les complexes familiaux, Lacan stabilisce un nesso fra un rapporto patologico con il cibo, riferendosi in particolare all'anoressia, e «una posizione del soggetto caratterizzata da una nostalgia incolmabile per l'Altro materno che sfiora l'attrazione oscura verso l'abisso della morte»; ${ }^{81}$ il vincolo familiare su cui si focalizza Lacan, individuando una relazione nostalgica con la figura materna, risulta in stretta connessione con l'archetipo del ritorno a casa. ${ }^{82}$

Tornando al concetto della nostalgia, il Dizionario dei temi distingue tra nostalgia «passiva, chiusa, regressiva»e nostalgia «aperta, creativa». Quest'ultima è resa possibile dall'accettazione della «finitudine come elemento costitutivo dell'esistenza», che contribuisce «a trasformare la nostalgia in linguaggio, in narrazione $\gg .{ }^{83}$

In effetti, la lingua tedesca, in cui la parola nostalgia è legata etimologicamente alla casa (Heimweb), prevede anche un'entrata lessicale che indichi una nostalgia di secondo grado, trasformata cioè in agency e in desiderio di viaggio (Fernweh).

${ }^{79}$ Ceserani, Domenichelli, Fasano, Dizionario dei temi letterari, pp. 1667-1668.

${ }^{80}$ Ivi, p. 1620.

${ }^{81}$ Massimo Recalcati, L'ultima cena: anoressia e bulimia, Milano, Mondadori, 1997, p. 37.

82 «The experience of weaning arouses in the infant a tendency toward death represented as the maternal image, a nostalgia for wholeness that can take many forms but always consists in a more or less sublimated return to the womb», Gilbert D. Chaitin, Lacan and the Object of Semiotics, in «SubStance», 57 (1988), 17.3, pp. 37-55, p. 46.

${ }^{83}$ Ceserani, Domenichelli, Fasano, Dizionario dei temi letterari, p. 1669. 
Una distinzione analoga si deve all'antropologo Vito Teti, che parla di nostalgia creativa in un intervento intitolato Antropologia dei migranti:

Occorre intercettare le tante nostalgie positive e creative che arrivano da chi è rimasto e da chi è partito. Da chi si trova a mezza parete. La nostalgia, nel senso di rapporto con i luoghi e le case, è una sorta di bene comune che andrebbe diversamente affrontata. La nostalgia, allora, non è per un passato di sete e di miseria, ma assume un valore utopico e di critica del presente ed è anche una nostalgia che tende a riprendere scarti, frammenti, schegge e raccogliere le briciole di un mondo scomparso, che però manda segnali di vita in un mondo sempre più piagato da catastrofi, guerre, carestie. Ho sempre distinto tra nostalgia patologica e nostalgia creativa; nostalgia regressiva e nostalgia critica; «nostalgia riflessiva» e «nostalgia restauratrice»: una ricorda il passato in modo retorico, l'altra agisce per recuperare tutto ciò che è salvabile. La nostalgia non neutralizza la storia ma, a certe condizioni, sprigiona dinamiche sovversive. Cercare l'utopia nel passato non significa essere nostalgici di una felicità perduta, ma rintracciare piccole isole di intimità nel mare della sofferenza. Il passato può e deve essere riscattato come un universo, un mondo sommerso, di potenzialità diverse, non compiute, suscettibili di future realizzazioni. Un riscatto, un risarcimento, una restituzione. Questa prospettiva invita a guardare con altro occhio luoghi, paesaggi, acque e cibi. ${ }^{84}$

Elementi tematici e stilistici come l'insistenza sui mezzi di trasporto, quindi sul macrotema del viaggio, e l'impiego orizzontale del futuro semplice, insieme al riscontro di posture enunciative caratterizzate dalla generosità con cui il soggetto lirico si rivolge al suo interlocutore, sembrano indicare nella produzione dei ventiquattro autori un atteggiamento generale di propensione verso il mondo.

Per tentare un'ipotesi, il meccanismo interno di questi testi poetici sarebbe la trasformazione della presa di consapevolezza della disgregazione del mondo in nostalgia creativa, per cui l'immaginario della morte, spesso legato a un'operazione di scavo, anche archeologico, nelle radici dell'identità collettiva è accompagnato da controspinte vitalistiche (non ultima, l'autoinvestitura poetica), in una sorta di disforia testuale: «L'ambivalenza della nostalgia può risolversi nella consapevolezza di un linguaggio che tenga aperto il rapporto col tempo $\gg .85$

Secondo Gianluigi Simonetti, una chiave di lettura della poesia contemporanea può essere individuata proprio in questo atteggiamento disforico, per esempio in autori come De Angelis o Salvia:

L'oscillazione tra fascino delle tenebre e richiamo alla chiarezza, in Salvia, sembra alludere a un'altra oscillazione, forte in lui e in altri suoi compagni, quella appunto tra

${ }^{84}$ Teti, Antropologia dei migranti, in La storia, le trasformazioni, p. 133.

${ }^{85}$ Ceserani, Domenichelli, Fasano, Dizionario dei temi letterari, p. 1669. 
mito delle origini e nevrosi della fine: il rinvio alla tradizione, anzi alle tradizioni, è ossessivo quanto quello al nuovo inizio. [...] Strade diverse, evidentemente, che forse s'incontrano in un punto solo, ma decisivo: il tentato recupero di meccanismi di comunicazione, intesa e interazione con il pubblico [...] la poesia si fa più progettualmente discorsiva. ${ }^{86}$

Simonetti si sofferma sulla produzione italiana degli anni Ottanta e Novanta, dove mito delle origini e nevrosi della fine sarebbero agenti in compresenza; autori come Patrizia Valduga, Gabriele Frasca, Mario Benedetti e Stefano Dal Bianco, per citarne alcuni fra quelli trattati dallo studioso, risultano, in accordo a un recente articolo di Riccardo Frolloni, le figure di riferimento più prossime alla generazione dei nati negli anni Ottanta e Novanta ( «la nostra stessa generazione [è] tutta intenta a fare i conti con i padri (e con gli zii) »). ${ }^{87}$ In questo senso, l'atteggiamento disforico riconosciuto nei testi di questi modelli potrebbe essere guardato come a un elemento di continuità, un'eredità attiva, insieme a quella «esigenza di comunicazione, riflessione e affabulazione (e quindi un rapporto più 'sano' e diretto col pubblico) $\gg^{88}$ che qui si è indicato nei termini di generosità interlocutoria.

In uno studio che guarda allo stesso arco temporale di Simonetti (vale a dire la «produzione compresa tra l'ultimo scorcio degli anni '70 e l'inizio degli anni '90»), ${ }^{89}$ Vincenzo Bagnoli, pur senza distinguere fra nostalgia passiva e nostalgia creativa, indica come chiave di lettura della poesia contemporanea proprio la trasformazione della funzione nostalgica: «Ciò che infatti sembra tramontare, o perlomeno trasformarsi radicalmente, è la funzione della nostalgia»; $9^{90}$ tale trasformazione risulta correlata a un diverso rapporto, sostanzialmente basato su un senso di «estraneità», con quello che il critico definisce «oggetto perduto». ${ }^{91}$ Nel quadro tracciato da Bagnoli, questo cambiamento si rifletterebbe nei testi nei termini di una discorsività ritrovata (o, per meglio dire, ricostruita) sul «rumore di fondo $\gg$ :

Nel momento in cui il già detto, il già stato pesano in modo gravissimo sull'espressione individuale, al lirico silenzio non può che subentrare il rumore di fondo, l'interferenza.

${ }^{86}$ Gianluigi Simonetti, La letteratura circostante: narrativa e poesia nell'Italia contemporanea, Bologna, il Mulino, 2018, pp. 163-197.

${ }^{87}$ Riccardo Frolloni, Ok boomer. Per una storia della poesia recente, su Le parole e le cose 2.

${ }^{88}$ Gianluigi Simonetti, Nuovi modi per andare a capo. Su alcune raccolte recenti di poesia italiana, in «Italianistica: Rivista di letteratura italiana», 37 (2008), 1, pp. 145-156, p. 146.

${ }^{89}$ Vincenzo Bagnoli, Contemporanea. La nuova poesia italiana verso il Duemila, Padova, Esedra, 1996, p. 13.

${ }^{90}$ Ibidem.

${ }^{91}$ Ivi, p. 37. 
[...] Tale ipotesi ha trovato ascolto tra i poeti contemporanei: la consapevolezza di una insufficienza del linguaggio oggi non può accontentarsi di una aperta rottura che porti al di fuori dei margini della comunicazione, e tenta anzi di lavorare sul rumore di fondo, ricomponendo un discorso. ${ }^{92}$

«Non parlarmi sto tentando | di capire qual è il suono», scrive Rafaiani; ${ }^{93}$ il soggetto lirico contemporaneo nasce alle prese con un rumore di sottofondo, che è anche il rumore fisiologicamente inarrestabile dell'epoca della riproducibilità tecnica. Come osserva ancora Simonetti: «La situazione [il rumore di sottofondo] chiede, o impone, di cambiare ritmi e parole usurati, o di parlare più forte, o di parlare più chiaro. E di rifondare la prima persona $\gg .{ }^{94}$

Questo gesto di rifondazione, discorsiva e progettuale, sembra alla base di quell'atteggiamento di propensione chiamato in causa fin dall'Introduzione del primo volume come «attivismo non contemplativo».95

Se prendiamo un archetipo noto come Nostalgia di Giuseppe Ungaretti, notiamo che lo stesso conglomerato tematico (la malattia, il sentimento di sradicamento, il canto) si risolve in una postura del soggetto lirico puramente contemplativa e, in ultima analisi, in un viaggio bloccato ( $\ll$ E come portati via $\mid$ si rimane»):

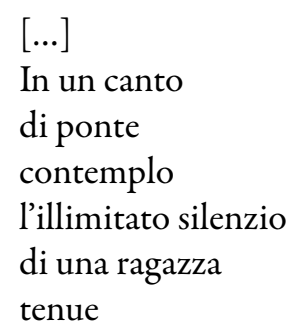

${ }^{92}$ Ivi, pp. 74-76.

${ }^{93}$ Mi viene incontro tutta uguale, vv. 5-6, Rafaiani, Poeti italiani, II, p. 172.

${ }^{94}$ Simonetti, La letteratura circostante, p. 196.

${ }^{95}$ «Qui si gioca, penso, lo scarto tra questi momenti verbali e il tempo poetico che li ha preceduti e generati: ora l'enunciazione dell'assenza diventa lotta contro l'assenza, attivismo non contemplativo, propensione. Cambia, rispetto a prima, che le rovine non vengono più (solo) contemplate. Parafrasando un titolo di Gadda, non I viaggi la morte - ma i viaggi anche se la morte. O meglio, i viaggi proprio perché la morte. È un movimento che costituisce già, almeno in parte, un arrivo: questo sforzo di apparato è motivato dall'aver qualcosa da dire - e infatti i referenti non scompaiono mai davanti, anzi, ci si rivolge loro con una generosità rara, spesso tenera, mai banale» (Martini, Introduzione a Poeti italiani, I, p. 6). 


\section{E come portati via}

si rimane ${ }^{96}$

La «nostalgia» presente nel testo di Ungaretti sembrerebbe quindi una nostalgia di primo grado, non ancora trasformata in spinta narrativa, propensione, agency.

Tra le poetiche contemporanee che, in misura variabile, costituiscono modelli di riferimento per quanti scrivono versi negli anni Venti del Duemila, quella di Patrizia Cavalli sembra enuclearsi da una sorta di nostalgia trasformata in spinta creatrice.

I temi dell'autoinvestitura e della percezione del gesto poetico come mestiere («Che forse non è questo il mio mestiere?») ${ }^{97}$ sono infatti costantemente presenti e rielaborati nella produzione cavalliana, dalla prima raccolta del 1974, pubblicata con Einaudi per volere di Elsa Morante, che ne scelse addirittura il titolo, Le mie poesie non cambieranno il mondo, fino all'ultima del 2020, Vita meravigliosa, in cui la stessa Morante interviene come personaggio di in un dialogo fittizio per ricongiungere il soggetto lirico a un ideale paradiso, che si direbbe perduto e ritrovato nel gesto poetico stesso (Con Elsa in Paradiso)..$^{98}$

In molti testi, infatti, il soggetto lirico cavalliano partecipa di un sentimento di sradicamento rispetto a una dimora originale da cui sarebbe stato, letteralmente, «mandato via»; così recita, per fare un esempio, L'Eden, nella prima sezione (Io li c'ero già stata) della raccolta del 2006, Pigre divinità e pigra sorte:

Mi hanno mandato via?

E io me lo rifaccio.

E visto che ci sono lo miglioro. ${ }^{99}$

Su questo tracciato, dalla circoscrizione della finitudine alla predisposizione, per cui il gesto poetico testimonia una sorta di preparare il canto da dentro, sembrerebbero muoversi anche i ventiquattro autori presi come campione, che, come osserva Paolo Giovannetti, sembrano confrontarsi «con un eccesso di autocoscienza espressiva». ${ }^{100}$ Per usare ancora le parole di Bagnoli, questi testi mostrerebbero «una rinnovata fiducia nei propri mezzi e nel senso del proprio fare.

${ }^{96}$ Nostalgia, Giuseppe Ungaretti, Vita d'un uomo. Tutte le poesie, Milano, Mondadori, 2009, p. 92.

${ }^{97}$ Che forse non è questo il mio mestiere?, Patrizia Cavalli, Pigre divinità e pigra sorte, Torino, Einaudi, 2006, p. 36.

${ }^{98}$ Cfr. Con Elsa in Paradiso, Patrizia Cavalli, Vita meravigliosa, Torino, Einaudi, 2020, pp. 15-17.

${ }^{99}$ L'Eden, Cavalli, Pigre divinità e pigra sorte, p. 15.

${ }^{100}$ Paolo Giovannetti, La poesia italiana degli anni Duemila. Un percorso di lettura, Roma, Carocci, 2017, p. 35. 
Questo senso si misura [...] soprattutto in un recupero della dimensione progettuale [...], intesa come esigenza di ricostruire. Questa è anzi la specificità che rende tali autori contemporanei a pieno titolo»: ${ }^{101}$

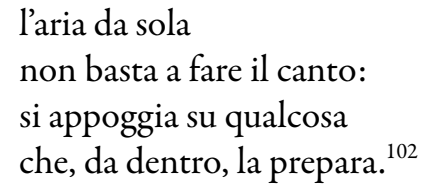

In altre parole, la postura di propensione a cui sembra richiamata la maggioranza dei testi raccolti nascerebbe proprio dalla trasformazione dell'osservazione nostalgica delle rovine in nostalgia creativa dell'enunciato, secondo la funzione della nevrosi della fine individuata da Simonetti:

Non solo molte delle parole più memorabili di queste poesie riguardano atti concreti l'esserci ora, il decidere oggi - che alludono a loro volta a una possibilità di intervento sul mondo [...] l'io lirico di Somiglianze si vede agire nel mondo, anche se non scorge una direzione, o forse soprattutto se non la scorge. ${ }^{103}$

È sempre dall'interno di questo flusso che chi scrive tenta di scorgere la direzione, senza scorgerla; oppure la direzione c'è ma l'occhio è malato, per rimandare anche allo studio di Niva Lorenzini Corpo e poesia nel Novecento italiano per cui, per esempio, in Nel condominio di carne di Valerio Magrelli, ${ }^{104}$ le parole trattano di malattie e di viaggi:

Distrutti i rapporti mente-corpo che presiedevano alla cartografia di Ora serrata retinae, ora si sezionano in parallelo carne-ossa-pelle-organi e parole che trattano di malattie, viaggi, memoria mineralizzata come traccia geologica interna al percorso

\footnotetext{
${ }^{101}$ Bagnoli, Contemporanea, p. 82. A questo proposito, Bagnoli parla nello specifico di una «ricomposizione del presente» (p. 69), rinviando a quella «estetica del presente» formulata da Giuseppe Patella in merito alla condizione postmoderna: «L'estetica del periodo della dissoluzione delle forme moderne dell'arte si muove ormai in una dimensione che non contempla più la rimemorazione o la nostalgia delle esperienze del passato, né in quella del "superamento" dialettico dell'estetica tradizionale; essa si vede invece completamente affidata ad un confronto reale ed efficace con la storia e in "presa diretta" con l'esistente, che le impone un radicale cambiamento di rotta. Contro una ormai perenta estetica dialettica e una fragile e fugace estetica per il presente, occorre dunque insistere su una più meditata e coerente estetica del presente, che nella pratica quotidiana ritrova le condizioni di una più concreta ed essenziale effettualità» (Giuseppe Patella, Sapere e potere, estetica e politica nell'età postmoderna: verso un postmodernismo della resistenza, in «Annali d'Italianistica», 9 (1991), pp. 92-105, p. 93).

102 si potrebbe entrare in te, vv. 9-12, De Gennaro, Poeti italiani, I, p. 138.

${ }^{103}$ Simonetti, La letteratura circostante, p. 166.

${ }^{104}$ Valerio Magrelli, Nel condominio di carne, Torino, Einaudi, 2003.
} 
biografico. E pare una mappatura di vicende, di accadimenti, che si sviluppano prima o dopo l'io, diventato anch'esso traccia ibridata tra migrazioni e innesti, invaso da viti e bulloni, occhiali e protesi di vario genere: un io dato in dimensione post-human, perfettamente rispondente al nostro presente globalizzato e scorporato. ${ }^{105}$

«La nostalgia è creativa», dice Natalia Ginzburg in un dialogo del 1990 con Marino Sinibaldi e Vittorio Foa:

NATALIA GINZBURG La nostalgia è creativa. È una parola screditata, oggi: ma è una parola invece nobile, e creativa

VITTORIO FOA Secondo me la nostalgia... Ci ho pensato molto: secondo me la nostalgia è un punto di partenza del futuro. Cioè tu ricordi perché hai bisogno di fare qualcosa che riguardi il futuro. Non è mai passività pura, la nostalgia

NATALIA GINZBURG Tu pensi?

VITTORIO FOA Ah, io sono convinto di questo! E questo proprio l'ho studiato in me stesso, a lungo. Quando avevo più acuta la nostalgia, vuol dire che avevo bisogno di fare qualcosa di nuovo

NATALIA GINZBURG Sì, qualcosa di vitale, non di mortifero

VITTORIO FOA Di vitale, ecco. Non pensi?

NATALIA GINZBURG È vero. L'hanno scambiata per qualcosa di mortifero, e si sono sbagliati

VITTORIO FOA Invece no! È una categoria assolutamente vitale. È bellissima la nostalgia: purché sia la nostalgia del futuro

NATALIA GINZBURG Purché sia la nostalgia del passato che vogliamo riallacciare a un futuro ${ }^{106}$

Caratteristica fondamentale della nostalgia creativa sembra infatti la capacità di tenere aperto il rapporto con il tempo (e quindi con l'interlocutore, con la narrazione), in una sorta di strabismo diacronico per cui il soggetto guarda contemporaneamente al passato (anche lontano) e al futuro (anche immediato).

${ }^{105}$ Niva Lorenzini, Corpo e poesia nel Novecento italiano, Milano, Mondadori, 2009, p. 119.

${ }^{106}$ Natalia Ginzburg, Ė difficile parlare di sé. Conversazione a più voci condotta da Marino Sinibaldi, Torino, Einaudi, 1999, pp. 234-235. 
$\mathrm{Di}$ «nostalgia di un futuro» parla anche il soggetto poetico sanguinetiano:

e a Roberto dissi (al Norman,

ancora) come il prefigurante, lo spettrale sogno la forma assunta (talvolta) della nostalgia;

e dissi: il sogno (talvolta) è ripiegamento (ma

nell'apparenza); è (dissi) nostalgia, ma delusa nostalgia

di un futuro; $;^{107}$

Lo stesso Sanguineti, in un intervento su «Paese Sera» del 1975, obietta alcune considerazioni di Italo Calvino sul tema del «dimenticare il futuro»nell'Odissea, considerazioni che mettevano in rapporto il nostos di Ulisse alla necessità di tenere insieme «l'impronta del passato e il progetto del futuro». ${ }^{108}$ Per Sanguineti invece, il futuro che Ulisse ha davanti non è altro che il suo passato: «l'Ulisse omerico approda al recupero del suo passato come un presente: la sua saggezza è la Ripetizione.$^{109} \mathrm{~A}$ sua volta, Calvino replica che «la desiderabilità d'un futuro da conquistare viene garantita dalla memoria d'un passato perduto $\gg .{ }^{110}$

In linea con la tesi calviniana, anche Emilia Di Rocco parla del nostos come un racconto salvato dalla nostalgia, ma anche della «nostalgia aperta» per le avventure future a cui il reduce rinuncia:

Lontano dalla sua terra d'origine, Odisseo non solo conduce un'esistenza da estraneo ma, come chi è in esilio, va incontro al rischio dell'oblio, l'esatto opposto della casa. È un pericolo dal quale, tuttavia, il desiderio di Penelope - la nostalgia - salva il Laerziade, nella misura in cui chi scorda il ritorno dimentica la casa e la strada che ad essa conduce. [...] Il reduce rinuncia alla nostalgia aperta dell'infinito e del mondo meraviglioso dei viaggi avventurosi, nel quale, pur essendo sempre presente come prospettiva ultima, il ritorno è continuamente rimandato. ${ }^{111}$

107 Purgatorio de l'Inferno, 3, Edoardo Sanguineti, Mikrokosmos. Poesie 1951-2004, Milano, Feltrinelli, 2004, p. 34.

108 «Ciò che Ulisse salva dal loto, dalle droghe di Circe, dal canto delle Sirene, non è solo il passato o il futuro. La memoria conta veramente - per gli individui, le collettività, le civiltà - solo se tiene insieme l'impronta del passato e il progetto del futuro» (Italo Calvino, Perché leggere i classici, Milano, Mondadori, 1995, p. 16).

${ }^{109}$ Ibidem.

${ }^{110}$ Ivi, p. 17.

${ }^{111} \mathrm{Di}$ Rocco, Raccontare il ritorno, pp. 58-64. Ma cfr. anche, della stessa, Baciare la terra. Un topos letterario, Roma, Carocci, 2012, che si conclude: «Il bacio alla terra diventa così espressione del desiderio dell'esule di tornare nel grembo ctonio della terra al momento della morte. La nostalgia della patria diventa desiderio latente del ritorno alla Grande Madre», p. 134. 
Un'istanza comune nei testi poetici analizzati afferisce proprio a questo strabismo cronico, a questa temporalità aperta. È la compresenza delle macerie e del futuro semplice, delle caverne e dei mezzi di trasporto, dell'operazione di scavo e della predisposizione alla partenza: «per questo non giungo, ma dico. per questo in un solo momento. nella gravità della vita, nella più incessante scrittura. | eredità io ti ho generato $\gg .{ }^{112}$

In ultima analisi, nella chiave di lettura della nostalgia creativa sembrerebbero riunirsi e dialogare programmaticamente i temi e gli oggetti testuali che ricorrono orizzontalmente nei ventiquattro autori raccolti: il nostos impossibile si trasforma da malattia in narrazione aperta e propensione all'agency, attraverso la testimonianza attiva delle rovine della cellula sociale. Va da sé che questa propensione all'agency dovrà essere vagliata da ulteriori studi e interventi che tengano conto anche di un'estensione del corpus autoriale, necessariamente cursorio, qui proposto.

A questo scopo, è in corso un approfondimento sulle forme di immaginario sociale e rappresentazioni del pubblico nei testi poetici degli anni Duemila, a partire dalla produzione dei nati negli anni Ottanta e Novanta. Il sentimento del destinatario (inteso nella postura del soggetto-poeta all'interno dei componimenti in cui il personaggio che dice «io» si posiziona come artista, problematizzando il suo gesto in relazione alla ricezione di una platea più o meno ampia di ascoltatori) che viene a prefigurarsi in questi campioni testuali sembra riconducibile, ancora una volta, alla funzione della nostalgia creativa: in molti dei casi analizzati infatti, la rappresentazione del pubblico sembra rispondere all'esigenza di costruire un ostacolo (per fare un esempio, in un testo di Guarino già citato, il «Poeta» deve adoperarsi per «zittire il pubblico insensibile»), ${ }^{113}$ che finisce per mettere in risalto il fatto che il soggetto parlante è detentore di un messaggio.

Per chiudere sulle parole di Armando Massarenti in una recente prefazione antologica, «I poeti ci sono. Sono molti e hanno molto da dire. Sono consapevoli della tradizione cui appartengono, ma anche fieri di far udire la propria voce unica e attuale. Dispongono di capacità di approfondimento, di sguardo critico, di una lingua riformata». ${ }^{114}$

${ }^{112}$ un diagramma cosi, Corsi, Poeti italiani, II, p. 45.

${ }^{113}$ Tutto era già pronto per il reading di poesie, v. 15, Guarino, Poeti italiani, I, p. 17.

${ }^{114}$ Armando Massarenti, Prefazione a Poesia d'oggi. Un'antologia italiana, a cura di Paolo Febbraro, pp. 7-11, Roma, Elliot, 2016, cit. p. 8. 


\section{Bibliografia}

Vincenzo Bagnoli, Contemporanea. La nuova poesia italiana verso il Duemila, Padova, Esedra, 1996.

Italo Calvino, Perché leggere i classici, Milano, Mondadori, 1995.

Patrizia Cavalli, Pigre divinità e pigra sorte, Torino, Einaudi, 2006.

Patrizia Cavalli, Vita meravigliosa, Torino, Einaudi, 2020.

Remo Ceserani, Mario Domenichelli, Pino Fasano, Dizionario dei temi letterari, vol. II, Milano, Garzanti, 2007.

Gilbert D. Chaitin, Lacan and the Object of Semiotics, in «SubStance», 57 (1988), 17.3, pp. 37-55.

Emilia Di Rocco, Baciare la terra. Un topos letterario, Roma, Carocci, 2012.

Emilia Di Rocco, Raccontare il ritorno. Temi e trame della letteratura, Bologna, il Mulino, 2017.

Natalia Ginzburg, È difficile parlare di sé. Conversazione a più voce condotta da Marino Sinibaldi, Torino, Einaudi, 1999.

Evelyn Grossman, La Creativité de la crise, Paris, Les Éditions de Minuit, 2020.

Niva Lorenzini, Corpo epoesia nel Novecento italiano, Milano, Mondadori, 2009.

Valerio Magrelli, Nel condominio di carne, Torino, Einaudi, 2003.

Giulia Martini (a cura di), Poeti italiani nati negli anni '80 e'90, Latiano, Interno Poesia, I vol. 2019; II vol. 2020.

Armando Massarenti, Prefazione a Poesia d'oggi. Un'antologia italiana, a cura di Paolo Febbraro, pp. 7-11, Roma, Elliot, 2016.

Paolo Giovannetti, La poesia italiana degli anni Duemila. Un percorso di lettura, Roma, Carocci, 2017.

Giuseppe Patella, Sapere e potere, estetica e politica nell'età postmoderna: verso un postmodernismo della resistenza, in «Annali d'Italianistica», 9 (1991), pp. 92105.

Massimo Recalcati, L'ultima cena: anoressia e bulimia, Milano, Mondadori, 1997.

Edoardo Sanguineti, Mikrokosmos. Poesie 1951-2004, Milano, Feltrinelli, 2004.

Gianluigi Simonetti, Nuovi modi per andare a capo. Su alcune raccolte recenti di poesia italiana, in «Italianistica: Rivista di letteratura italiana», 37 (2008), 1, 
pp. 145-156.

Gianluigi Simonetti, La letteratura circostante: narrativa e poesia nell'Italia contemporanea, Bologna, il Mulino, 2018.

Vito Teti, Antropologia dei migranti, in La storia, le trasformazioni. Piero Bevilacqua e la critica del presente, a cura di Leandra D'Antone e Marta Petrusewicz, Roma, Donzelli, 2015.

Giuseppe Ungaretti, Vita d'un uomo. Tutte le poesie, Milano, Mondadori, 2009.

\section{Sitografia}

Riccardo Frolloni, Ok boomer. Per una storia della poesia recente, su Le parole e le $\operatorname{cose}^{2}$ (10 dicembre 2020)

$<$ http://www.leparoleelecose.it/?p=40112> 